\title{
The Different Therapeutic Choices with ARBs. Which One to Give? When? Why?
}

\author{
Csaba András Dézsi ${ }^{1}$
}

Published online: 3 March 2016

(C) The Author(s) 2016. This article is published with open access at Springerlink.com

\begin{abstract}
The renin-angiotensin-aldosterone system plays an important role in the pathophysiology of hypertension and is closely related with cardio- and cerebrovascular events and chronic kidney diseases. Each angiotensin receptor blocker (ARB) is important in the treatment of hypertension, according to the results of recent years. This is a practical review of the available evidence on the different benefits of ARBs beyond their blood pressure-lowering effect, with an emphasis on the differences found between the particular compounds and the therapeutic implications of the findings, with specific reference to the co-morbidities.
\end{abstract}

$\begin{array}{ll}\begin{array}{l}\text { Abbreviations } \\ \text { ACEI }\end{array} & \begin{array}{l}\text { Angiotensin converting enzyme } \\ \text { inhibitor }\end{array} \\ \text { ACTIVE-I } & \begin{array}{l}\text { Atrial Fibrillation Clopidogrel Trial } \\ \text { With Irbesartan for Prevention of }\end{array} \\ & \text { Vascular Events } \\ \text { AD } & \text { Alzheimer's disease } \\ \text { AF } & \text { Atrial fibrillation } \\ \text { AMADEO } & \text { A comparison of telMisartan versus } \\ & \text { losArtan in hypertensive type 2 DiabEtic } \\ \text { patients with Overt nephropathy } \\ \text { ATB } 2 & \text { Angiotensin receptor blocker } \\ \text { ATHLETE } & \text { Angiotensin II Type 2 } \\ & \text { ARB trial of hypertension in obese } \\ & \text { patients with hyperinsulinemia assessed } \\ \text { by oral glucose tolerance test }\end{array}$

Csaba András Dézsi

dcsa62@gmail.com

1 Department of Cardiology, Petz Aladár County Teaching Hospital, Gyor, Hungary
CALM

CHARM

CKD

$\mathrm{CV}$

CVD

DETAIL

DM

ED

ELITE

eNOS

ESRD

GISSI-AF

GMP

HbA1c

$\mathrm{HF}$

HOMA-IR

IDNT

INNOVATION

IR

K-MetS

LIFE
Candesartan And Lisinopril

Microalbuminuria study

Candesartan in Heart Failure-

Assessment of Reduction in Mortality and Morbidity

Chronic kidney disease

Cardiovascular

Cardiovascular disease

Diabetes Exposed to Telmisartan And enalaprIL

Diabetes mellitus

Erectile dysfunction

Evaluation of Losartan In The Elderly

Endothelial Nitric Oxide Synthase

End-stage renal disease

Gruppo Italiano per lo Studio della Sopravvivenza nell'Infarto MiocardicoAtrial Fibrillation

Guanosine Monophosphate

Hemoglobin A1c:

Glycosylated Hemoglobin

Heart failure

Homeostasis model assessment of insulin resistance

Irbesartan Diabetic Nephropathy Trial

The INcipieNt to OVert: Angiotensin II receptor blocker, Telmisartan,

Investigation On type 2

diabetic Nephropathy

Insulin resistance

The Efficacy of Fimasartan for Cardiovascular Events and Metabolic Syndrome

Losartan Intervention For Endpoint reduction 
MARVAL

MI

MMSE

MOSES

mRNA

nNOS

NYHA

NYHA-FC

ONTARGET

ORIENT

OSCAR

PPAR- $\gamma$

PRoFESS

RAAS

RENAAL

RESOLVD

ROADMAP

RR

Safe-KanArb

SARTAN-AD

SCOPE

SPECT

SUA

TGF- $\beta$

TRANSCEND

Val-HeFT

VALIANT
MicroAlbuminuria Reduction With

VALsartan

Myocardial infarction

Mini-Mental State Examination

The MOrbidity and Mortality after Stroke-Eprosartan compared with nitrendipine in Secondary prevention study

Messenger Ribonucleic Acid

Neuronal Nitric Oxide Synthase

New York Heart Association

New York Heart Association Functional Classification

Ongoing Telmisartan Alone and in Combination With Ramipril Global End Point Trial

Olmesartan Reducing Incidence of End

Stage Renal Disease in Diabetic

Nephropathy Trial

The OlmeSartan and Calcium

Antagonists Randomized Study

Peroxisome Proliferator-activated

Receptor Gamma

The Prevention Regimen for Effectively

Avoiding Second Strokes

Renin-angiotensin-aldosterone system

Reduction of Endpoints in NIDDM with the Angiotensin II Antagonist Losartan Study

Randomized Evaluation of Strategies for Left Ventricular Dysfunction

Randomized Olmesartan and Diabetes Microalbuminuria Prevention Study

Relative Risk

Safety and Efficacy of Fimasartan in Patients with Arterial Hypertension

A randomized, open label, proof of concept study of Telmisartan vs. Perindopril in hypertensive mildmoderate Alzheimer's disease patients

Study on Cognition and Prognosis in the Elderly

Single-photon emission computed tomography

Serum uric acid

Transforming Growth Factor Beta

Telmisartan Randomized Assessment Study in ACE-Intolerant Subjects With Cardiovascular Disease Trial

Valsartan Heart Failure Trial

VALsartan In Acute myocardial

iNfarcTion
VALUE

VF-HT-AF

The Valsartan Antihypertensive Longterm Use Evaluation

Valsartan and Fluvastatin on

Hypertensive patients with non-permanent Atrial Fibrillation

VIVALDI study to inVestIgate the efficacy of telmIsartan versus VALsartan in hypertensive type 2 DIabetic patients with overt nephropathy

URAT Renal uric acid transporter

\section{Key Points}

Therapy customized for individuals is extremely important, especially for patients with high blood pressure who are increased risk of end organ damage.

When initiating angiotensin receptor blocker treatment, it is recommended to assess the clinical effects, which are proven to be divergent today, of each agent and their indications in light of the comorbidities.

\section{Introduction}

The appearance of angiotensin receptor blockers (ARBs) amongst the therapeutic options in the treatment of cardiovascular diseases (CVDs) was a new milestone in the history of hypertension treatment. It further widened the range of possibilities for personalized therapy, especially for patients who cannot tolerate the use of angiotensin converting enzyme inhibitors (ACEIs). ARBs have shown excellent efficacy, they have no negative metabolic effects and they cause no accumulation of bradykinin. They also have an ability to activate the angiotensin II type $2\left(\mathrm{AT}_{2}\right)$ receptors, which causes vasodilatation in the small vessels and presumably leads to additional cardiac and renal protection.

There is a vast amount of literature on antihypertensive and cardiovascular (CV) therapeutic choices, and recommendations are available as to when a renin-angiotensinaldosterone system (RAAS) inhibitor should be the first drug of choice. According to the latest international guidelines, ACEIs or ARBs should be preferred in patients with co-morbid microalbuminuria, renal dysfunction and chronic kidney disease (CKD), metabolic syndrome and diabetes mellitus (DM), atherosclerosis, chronic stable angina and previous myocardial infarction (MI), atrial fibrillation (AF) as well as heart failure (HF) [1-3]. 
As to which particular ARB should be preferred in case of different co-morbid conditions, no guidance is available. Also, there is a lack of substantial direct comparative trials between different ARBs regarding their effects beyond blood pressure lowering. Nevertheless, several studies are available where certain ARBs have shown additional beneficial effect; the present review of the available evidence should provide help in treatment selection for individual patients.

A comprehensive PubMed search was performed in August 2015, using the key words "angiotensin receptor blocker," “azilsartan," “candesartan," “eprosartan," “irbesartan," “losartan," “olmesartan," “telmisartan," "valsartan" and "fimasartan," identifying relevant articles concerning the efficacy of ARBs in clinical conditions beyond hypertension. References of identified articles were also searched for relevant articles (Tables 1, 2).

\section{Prevention of Cardiovascular Events and Mortality}

CVDs account for about $30 \%$ of all deaths in the world, ischemic heart diseases and stroke being responsible for the majority (4/5) of them. Underlying atherosclerosis can be found in around $75 \%$ of death cases due to CVDs [4].

The selective inhibition of angiotensin II on $\mathrm{AT}_{1}$ receptors blocks the systemic effects of the RAAS, including vasoconstriction, stimulation of aldosterone synthesis and renal absorption of sodium. Furthermore, $\mathrm{AT}_{1}$ inhibition reduces cardiac and vascular oxidative stress, inflammation and remodeling, thus improving endothelial dysfunction. The decrease of RAAS-related vascular inflammation may prevent the development of atherosclerosis, consequently reducing the risk of major CV events [4-6].
The blockade of $\mathrm{AT}_{1}$ receptors also results in increased angiotensin II activity on $\mathrm{AT}_{2}$ receptors, leading to vasodilation and natriuresis through bradykinin, nitric oxide, prostaglandin and cyclic guanosine monophosphate (GMP) pathways, generally showing an opposite effect to the action of $\mathrm{AT}_{1}$ receptors. Thus selective blockade of $\mathrm{AT}_{1}$ receptors can contribute to additional $\mathrm{CV}$ protection of ARBs [4, 7].

The primary goal of today's therapeutic strategy for CVDs is to control and decrease the existing risk factors and consequently decrease the occurrence of $\mathrm{CV}$ events and consequent morbidity and mortality. The designs of several recent clinical trials reflect this approach, investigating the reduction of $\mathrm{CV}$ events as trial endpoints. ARBs have shown the ability to reduce the risk of stroke and HF as well as the risk of major $\mathrm{CV}$ events in prospective randomized trials [8].

\subsection{Cardiovascular Protection}

Telmisartan is the only ARB indicated for the reduction of $\mathrm{CV}$ morbidity in patients with manifest atherothrombotic CVD, based on the results of the ONTARGET study [9]. It has shown a similar reduction in the composite endpoint of CV death, MI, stroke, or hospitalization due to HF to that of the active comparator ramipril [10]. The TRANSCEND study, while it failed to reach the composite primary endpoint, showed that telmisartan did reduce hospitalizations for CV reasons, and left ventricular hypertrophy, and fewer patients had the combination of macrovascular and microvascular events plus microalbuminuria [11]. In addition, a combined analysis with data from PRoFESS showed a significant benefit of telmisartan on CV death as well as MI and stroke [12].

Losartan has also shown benefits in reducing the relative risk of the composite endpoint of death, MI or stroke by

Table 1 Currently approved indications of ARBs in the US [123] and EU [10]

\begin{tabular}{|c|c|c|c|c|c|c|}
\hline ARBs & Hypertension & $\begin{array}{l}\text { Heart } \\
\text { failure }\end{array}$ & $\begin{array}{l}\text { Diabetic } \\
\text { nephropathy }\end{array}$ & $\begin{array}{l}\text { Prevention } \\
\text { of stroke }\end{array}$ & $\begin{array}{l}\text { Cardiovascular risk } \\
\text { reduction }\end{array}$ & $\begin{array}{l}\text { Following myocardial } \\
\text { infarction }\end{array}$ \\
\hline Azilsartan & $\mathrm{x}$ & & & & & \\
\hline Candesartan & $\mathrm{x}$ & $\mathrm{x}$ & & & & \\
\hline Eprosartan & $\mathrm{x}$ & & & & & \\
\hline Irbesartan & $\mathrm{x}$ & & $\mathrm{x}$ & & & \\
\hline Losartan & $\mathrm{x}$ & $\mathrm{x}^{\mathrm{a}, \mathrm{b}}$ & $\mathrm{x}$ & $\mathrm{x}$ & & \\
\hline Olmesartan & $\mathrm{x}$ & & & & & \\
\hline Telmisartan & $\mathrm{x}$ & & & & $\mathrm{x}$ & \\
\hline Valsartan & $\mathrm{x}$ & $x^{a}$ & & & & $\mathrm{x}$ \\
\hline
\end{tabular}

$A C E$ angiotensin converting enzyme, $A R B$ angiotensin receptor blocker

${ }^{\text {a }}$ In patients unable to take ACE inhibitors

b Only in the EU 
Table 2 Summary conclusions

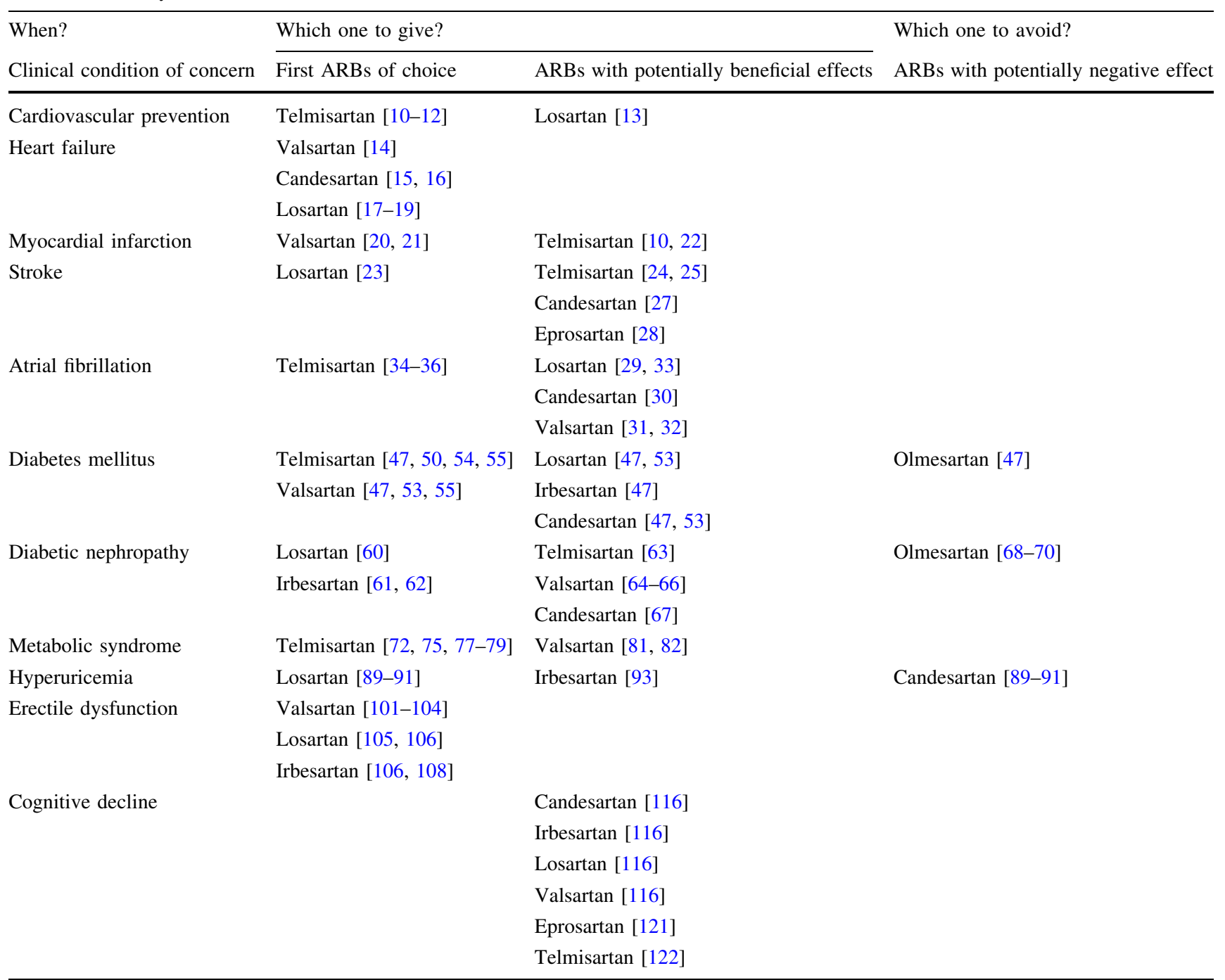

$A R B$ angiotensin receptor blocker

$13 \%$ compared with atenolol in the LIFE study, with more benefit amongst diabetic patients, where mortality was reduced by $39 \%$ [13].

\subsection{Heart Failure}

Valsartan, candesartan and losartan are indicated for the second-line treatment of HF in cases of ACEI intolerance [9].

In HF patients not receiving ACEIs, valsartan reduced all-cause mortality by $33 \%$ and composite mortality and morbidity risk by $44 \%$ compared with placebo in the ValHeFT trial. In the overall study population, valsartan caused significant improvements in New York heart association (NYHA) class, ejection fraction, and HF signs and symptoms and reduced the rate of hospitalization [14].
Candesartan had an effect similar to that of enalapril on left ventricular function and New York heart association functional classification (NYHA-FC) in the RESOLVD study [15]. The consequent CHARM trial showed that candesartan also caused a significant reduction in the risk of $\mathrm{CV}$ deaths and hospital admissions for HF compared with placebo [16].

The ELITE I and II trials have both shown that in elderly HF patients, treatment with losartan was similar to that with captopril in terms of all-cause mortality, sudden death or resuscitated arrests as well as NYHA class improvement. In the first ELITE study, there was also a lower mortality found with losartan, primarily due to a greater reduction in sudden cardiac death [17, 18]. It seems that the use of $150 \mathrm{mg}$ losartan has additional benefits to those of the 50-mg dose, as it reduced the rate of death or admission for HF and left ventricular ejection fraction [19]. 


\subsection{Myocardial Infarction}

Valsartan is also indicated for the treatment of patients with HF or asymptomatic left ventricular systolic dysfunction after a recent MI [9]. Treatment with valsartan resulted in similar changes in cardiac volume, ejection fraction, and infarct segment length between baseline and 20 months after MI compared with ramipril in the VALIANT trial. Valsartan was non-inferior to captopril in terms of total mortality and CV death, MI and HF [20]. Valsartan also showed long-term benefits when administered prior to percutaneous coronary intervention, resulting in a significantly reduced ratio of major adverse CV events [21].

The use of telmisartan was associated with a significant reduction in the number of MIs in hypertensive patients during the TRANSCEND trial compared with normotensive individuals [22].

In comparison with ramipril, telmisartan use resulted in a similar incidence of MI in patients with vascular disease of high-risk diabetes [10].

\subsection{Stroke}

Losartan is also indicated for hypertensive patients at risk of stroke [9]. In the LIFE study, losartan reduced the risk of any stroke, fatal stroke and atherothrombotic stroke significantly more than did atenolol (by 40,70 and $45 \%$, respectively) [23].

Telmisartan showed a nonsignificant reduction of recurrent stroke versus placebo in the PRoFESS trial; according to a post hoc analysis, from 6 months, the reduction of the number of strokes was significant [24, 25].

In elderly patients with isolated systolic hypertension, candesartan treatment resulted in a significant relative risk reduction of all stroke events in comparison with other antihypertensive treatment [26]. Candesartan also showed beneficial effects used as a 7-day course following an acute ischemic stroke, significantly improving CV morbidity and mortality [27].

The MOSES trial, investigating high-risk hypertensive stroke patients, showed that compared to nitrendipine, eprosartan therapy resulted in significantly fewer cerebrovascular events during the 2.5 years of follow-up [28].

\subsection{Atrial Fibrillation}

The use of losartan, valsartan and candesartan has been associated with a reduced incidence of new-onset AF, according to post hoc analyses of the LIFE, VALUE, CHARM and Val-HeFT trials, with a relative risk reduction of 20-35\% in new-onset cases [29-32]. Losartan was also found to suppress the maximum duration and the total duration of paroxysmal AF in patients with sick sinus syndrome without causing any significant hemodynamic changes [33].

Telmisartan significantly reduced the recurrence of $\mathrm{AF}$ compared with carvedilol, amlodipine as well as ramipril [34-36].

Studies with recurrent AF with other ARBs are slightly conflicting. Fogari et al. [37, 38] have found both valsartan and losartan to prevent new occurrences of AF compared with amlodipine. However, the GISSI-AF Investigators could not confirm the preventive role of valsartan in patients who already had AF in their history [39, 40]. Similarly, although irbesartan showed a significant reduction of recurrent AF in the study by Madrid et al. [41] in patients with persistent $\mathrm{AF}$, there was no difference regarding recurrent $\mathrm{AF}$ between irbesartan and placebo in the ACTIVE-I trial; irbesartan reduced neither the risk of hospitalization for $\mathrm{AF}$ nor the risk of cardioversion [41, 42].

In the case of valsartan, the VF-HT-AF study has been designed and will probably shed more light on the benefits of this drug in the upstream therapy of recurrent $\mathrm{AF}$ in hypertensive patients [43].

\section{Prevention of Diabetes Mellitus and Associated Risk Factors}

The occurrence of hypertension is twice as common in diabetic patients as in the non-diabetic population. DM is a general CV risk factor; it causes elevated susceptibility to atherosclerosis, and it is an independent predictor of numerous adverse outcomes, including HF, stroke and peripheral vascular disease. Adequate glycemic as well as blood pressure control are therefore essential in the treatment of diabetic patients and those at risk of DM [4].

RAAS inhibitors have been found to improve insulin sensitivity. The anti-diabetic properties of ARBs may also include activation of Peroxisome proliferator-activated receptor gamma (PPAR- $\gamma$ ), augmentation of blood flow to muscles and upregulation of glucose transporter expression in muscle, suppression of oxidative stress and anti-inflammatory action, inhibition of fibrosis through inhibition of transforming growth factor beta (TGF- $\beta$ ) as well as enhancement and modulation of insulin signaling [44-47]. The novel compound azilsartan has also shown insulinsensitizing effects in obese rats, and clinical trials are on their way to assess the effect on human insulin resistance (IR) $[48,49]$.

According to a meta-analysis of eight trials, telmisartan was superior to other ARBs in reducing fasting plasma glucose and increasing adiponectin levels. Using an 80-mg dose, telmisartan may also reduce fasting plasma insulin levels as well as homeostasis model assessment of insulin resistance (HOMA-IR) [50]. 
ARB therapy can reduce the incidence of new onset diabetes compared with placebo in patients with high $\mathrm{CV}$ risk and/or hypertension [51, 52]. The meta-analysis of LIFE, SCOPE and VALUE trials has shown that losartan, candesartan as well as valsartan can cause a clinically significant decrease in the incidence of new onset diabetes, with a combined estimated relative risk (RR) of 0.80 for all the three agents [53]. Similarly, the meta-analysis of the TRANSCEND and PRoFESS trials has shown that treatment with telmisartan is associated with a $16 \%$ reduction in the risk of new onset DM compared with placebo [54].

These results are also supported by a population-based cohort of hypertensive patients, showing a similar protective effect for valsartan, irbesartan, candesartan and telmisartan, using losartan as a baseline reference; meanwhile, olmesartan use resulted in a slightly increased hazard ratio [47].

ARBs have also shown cardioprotective properties in patients with type $2 \mathrm{DM}$. In a population-based cohort study among older diabetic patients, telmisartan and valsartan (with an adjusted hazard ratio of 0.85 and 0.86 , respectively) were both associated with a lower risk of admission to hospital for acute MI, stroke or HF, compared with irbesartan. With respect to the same primary outcome, no differences were found between irbesartan, losartan or candesartan [55].

\section{Renal End-Organ Protection}

In patients with CKD, hemodynamic changes occur during the adaptation to functional tissue loss, regardless of the etiology of the renal failure. Stimulation of the RAAS increases intraglomerular pressure, leading to capillary damage, increased proteinuria and consequent interstitial inflammation and fibrosis. Proteinuria is an independent risk factor for end-stage renal disease (ESRD) and mortality; its reduction is associated with a decreased rate of glomerular filtration rate decline. Therefore both blood pressure control and reduction of proteinuria play a key role in CKD management in hypertensive patients with or without DM [56, 57].

According to a recent network meta-analysis, ARBs are the most effective antihypertensive agents for the prevention of ESRD, showing significant benefit compared with placebo [58]. In diabetic patients with nephropathy, administration of ARBs suppresses both oxidative stress and inflammation, patients with higher renal stress marker values benefiting the most in terms of oxidative stress marker and urinary albumin excretion rate reduction [59].

Compared with placebo, losartan given in addition to conventional antihypertensive treatment reduced the risk of ESRD by $28 \%$ and decreased the level of urinary protein excretion by $35 \%$ in patients with coexistent diabetic nephropathy in the RENAAL study [60].

In the IDNT trial, irbesartan reduced the risk of doubling serum creatinine levels by $33 \%$ and the onset of ESRD by $23 \%$ compared with placebo in hypertensive patients with nephropathy due to DM. Benefits of irbesartan use compared with amlodipine treatment were even more pronounced [61]. Higher doses of irbesartan also caused a sustained long-term reduction in urinary albumin excretion rate, even after withdrawal of all antihypertensive treatment [62].

A recent meta-analysis of 20 randomized controlled telmisartan trials (ONTARGET, TRANSCEND, DETAIL, INNOVATION, AMADEO and VIVALDI, among others) performed mainly in diabetic patients concluded that telmisartan therapy is likely effective in the improvement of proteinuria or in the prevention of its progression. Telmisartan caused a statistically significant reduction in percent changes of urinary albumin/protein excretion and urinary albumin/protein to creatinine ratio with telmisartan relative to other ARBs, ACEIs and other therapy by 20,14 and $40 \%$, respectively [63].

The addition of valsartan to conventional therapy significantly slowed the rate of renal function decline, reduced the doubling of serum creatinine levels and delayed the appearance of ESRD in hypertensive patients with advanced CKD compared with placebo [64]. In patients with type II diabetes and microalbuminuria with or without hypertension, valsartan significantly reduced elevated urine albumin excretion compared with baseline in the MARVAL trial. Valsartan also induced regression to normoalbuminuria in a significantly greater proportion of patients than amlodipine (29.9 vs. $15.5 \%$ ) [65]. Valsartan reduced albuminuria by more than 50 in $37.6 \%$ and returned to normal in $18.9 \%$ of the assessed hypertensive patients in an open-label observational study, with a more pronounced effect on those with DM than on the non-diabetic population [66].

Candesartan was shown to reduce urinary albumin/creatinine ratio by $30 \%$ in the CALM study [67].

Olmesartan was shown to increase the time to the onset of microalbuminuria in diabetic patients in the ROADMAP study. However, a higher rate of death from CV causes was observed in the olmesartan group than in the placebo group among patients with preexisting coronary heart disease [68]. CV death was also higher in the olmesartan group in the ORIENT trial investigating diabetic patients with overt nephropathy [69]. A retrospective cohort analysis of DM patients has also found that olmesartan use was associated with an adjusted hazard for all-cause hospitalization or allcause mortality of 1.11 in subjects with a history of CVD and 1.21 in subjects with CKD [70]. The increase found in the risk of fatal $\mathrm{CV}$ events might be attributable to the aggressive blood pressure lowering properties of 
olmesartan, or otherwise, the result of the so-called J-curve phenomenon [71].

\section{Obesity and Metabolic Syndrome}

Abdominal obesity is often associated with hypertension. Obese patients with hypertension are also more prone to developing diabetes than those who are normotensive. The severity of diseases related to obesity (such as DM or CVD) primarily correlates to body fat distribution. The negative relationship between adiponectin and adipose tissue is stronger with visceral fat rather than subcutaneous fat; visceral fat remodeling therefore can be beneficial in metabolic syndrome [72]. Hypoadiponectinemia is a key factor in metabolic syndrome. Adiponectin levels can be increased by insulin sensitizers and PPAR- $\gamma$ agonists, as PPAR- $\gamma$ may ameliorate the accumulation of visceral adipose tissues and sensitize insulin action [73].

One of the important features of drugs that suppress the RAAS is the absence of negative metabolic effects. Among antihypertensive drugs, thiazide diuretics and $\beta$-blockers enhance IR. Dihydropyridine calcium antagonists have no apparent effects on glucose metabolism and IR, while ACEIs and ARBs can enhance insulin sensitivity [74].

The PPAR- $\gamma$ agonist telmisartan improves both hemodynamic and metabolic abnormalities found in hypertensive patients with obesity. The results of a recent metaanalysis showed that telmisartan therapy significantly improves metabolic parameters such as fasting glucose, insulin, hemoglobin A1c: glycosylated hemoglobin (HbA1c) levels and HOMA index in patients with metabolic syndrome. Data for adiponectin levels were less robust but nevertheless showed significant improvement [75]. The ATHLETE study also showed that in obese patients with hypertension and IR, telmisartan significantly improved the hyperinsulin response to glucose loading [76]. Telmisartan treatment was further associated with improvement of vascular inflammation, reductions in visceral fat and serum TNF- $\alpha$ levels as well as increases in serum adiponectin in a number of studies [72, 77-79].

When compared with telmisartan, eprosartan, losartan or valsartan did not show the above detailed beneficial effects in obese hypertensive subjects [72, 78, 80]. However, in obese but otherwise healthy subjects, valsartan did decrease fasting plasma insulin levels, and in patients with impaired glucose metabolism, valsartan treatment reduced abdominal subcutaneous adipocyte size as well as adipose tissue macrophage infiltration markers, and increased fasting and postprandial adipose tissue blood flow compared with placebo [81, 82].

Although it has not yet been supported by clinical evidence, fimasartan, the ninth ARB, may also be mentioned here. It was found to have a successful antihypertensive efficacy and safety profile in Safe-KanArb [83], which is a large cross-sectional population study in South Korea. Furthermore, the same results were observed in a recent study conducted on a low-to-moderate risk hypertension population in Mexico [84]. Currently, treating high blood pressure is the sole indication for it use in the aforementioned countries. However, the results of the K-MetS study [85], which are expected to be released in 2016, aside from providing information on the antihypertensive efficacy of fimasartan-based treatment, are going to provide valuable information concerning other potential effects detected in patients with metabolic syndrome in case of such treatments.

\section{Hyperuricemia}

Several observations indicate that hyperuricemia is an independent risk factor for CVD. Raised serum uric acid (SUA) levels are found in approximately $25 \%$ of hypertensive patients; SUA may further be increased by the use of loop and thiazide diuretics $[86,87]$. The renal uric acid transporter (URAT1) was shown to be involved in the effect of ARBs on SUA level. Losartan and pratosartan have been found to be potent inhibitors of URAT1. However, there is a lack of inhibitory effect of candesartan, olmesartan and valsartan on URAT1, whereas a transstimulation of URAT1 by these ARBs was found at higher concentrations. Such trans-stimulatory effects may lead to an increase of SUA level [88].

Telmisartan exhibited only a cis-inhibitory effect but not a trans-stimulatory effect on URAT1. However, urinary excretion of telmisartan is less than $0.02 \%$; therefore no uricosuric effect of telmisartan can be observed clinically [88].

Losartan increased the excretion of uric acid and decreased the SUA level in both healthy and hypertensive subjects. Candesartan, however, was found to slightly but significantly increase uric acid levels [89-91]. Eprosartan had no effect on SUA concentrations or urine uric acid excretion in patients with mild-to-moderate essential hypertension [92]. Lately, irbesartan treatment was also found to be associated with the decrease of SUA levels, but only in patients with higher baseline SUA values [93].

\section{Erectile Dysfunction}

Erectile dysfunction (ED) seems to be strongly correlated with hypertension, and is also considered an early predictor of silent coronary heart disease. ED was found to be a potent predictor of all-cause death and the composite of $\mathrm{CV}$ death, MI, stroke and HF in men with CVD in the 
ONTARGET/TRANSCEND trials. Also, older CV drugs such as $\beta$-blockers or some diuretics seem to have a deteriorating effect on erectile function [94, 95]. Penile cavernous smooth muscle tone is partially balanced by bradykinin-induced relaxation and angiotensin II-induced contraction. Since the tissue and plasma levels of both peptides are regulated by the RAAS system, its inhibitors would be a prudent choice in the treatment of hypertension in patients with ED [96].

Several preclinical and clinical studies have shown beneficial effects of some ARBs on sexual function; however, not all studies have shown significant results compared with placebo $[97,98]$. In mice, irbesartan was shown to improve penile endothelial function by reduction of vascular and cavernosal oxidative stress, while olmesartan improved the malondialdehyde concentrations and increased messenger ribonucleic acid (mRNA) levels of endothelial nitric oxide synthase (eNOS) and neuronal nitric oxide synthase (nNOS) in the penis [99, 100].

In large prospective studies, the use of valsartan increased the rate of sexual intercourses per week and caused a reduction in ED, with improved orgasmic function, intercourse and overall sexual satisfaction in hypertensive men [101, 102]. In comparative studies, long-term use of valsartan was also shown to significantly improve sexual activity in comparison with the $\beta$-blockers carvedilol and atenolol, with the latter also reducing serum testosterone levels [103, 104].

Treatment with losartan improved erectile function, sexual satisfaction and frequency of sexual activity in hypertensive patients. Losartan alone or in combination with tadalafil significantly improved ED in diabetic patients, those with mild to moderate ED profiting the most from its use [105, 106].

In hypertensive patients with the metabolic syndrome, treatment with irbesartan alone or in combination with hydrochlorothiazide was associated with an improvement of sexual desire, frequency of sexual contacts and erectile function. Irbesartan also improved erectile function recovery in prostatectomized patients [107, 108].

Treatment with telmisartan did not significantly improve or worsen ED in the ONTARGET/TRANSCEND studies [94].

\section{The Future of Angiotensin Receptor Blockers: Protection of Cognitive Function}

The human brain is extremely dependent on proper functioning of its vascular system. There is ample evidence supporting the causal relationship of vascular dysfunction and hypertension with the development of dementia and the decline of cognitive function [109-113]. Furthermore, hypertension also seems to increase the risk and progression of Alzheimer's disease (AD) [114, 115].

According to the so-called $\mathrm{AT}_{2}$ hypothesis, parallel to the selective blockade of $\mathrm{AT}_{1}$ receptors during $\mathrm{ARB}$ use, $\mathrm{AT}_{2}$ is activated and contributes to some protective effects of ARBs. These protective qualities distinguish them from ACEIs, for which such modulator effects are not present. In the brain, the activation of $\mathrm{AT}_{2}$ decreases inflammation, superoxide production and axon degeneration, promotes neuronal cell differentiation and leads to activation of the repair systems [116].

Several animal studies have shown the benefits of ARBs on cognitive function. In adult and also aged mice, losartan exhibited protection against the onset of cognitive dysfunction, even despite high levels of soluble $A \beta$ species and $A \beta$ plaque load. Losartan also rescued evoked arterial, hemodynamic and neurometabolic responses even at an advanced pathological stage. Also, selective downregulation of $\mathrm{AT}_{1}$ and restoration of $\mathrm{AT}_{4}$ receptor levels were observed [117]. In hypertensive rats, olmesartan treatment restored the cognitive decline and ameliorated leakage from brain microvessels. Olmesartan also decreased brain ATII levels [118].

Telmisartan seems to protect against hypertension-associated cognitive decline not only by its $\mathrm{AT}_{1}$ receptor blockade, but also partly because of its PPAR- $\gamma$ activation in the hippocampus. Co-administration of a PPAR- $\gamma$ antagonist with telmisartan partially attenuated the protective effect of telmisartan on cognitive decline [119]. Telmisartan was also shown to significantly restore cognitive functions impaired by chronic stress and decrease forgetfulness [120].

In humans, ARBs have been shown to be associated with a significant reduction in the incidence and progression of AD as well as dementia compared with ACEIs or other CV drugs in a robust prospective cohort study. Dose response effects were studied and could be observed on candesartan, irbesartan, losartan and valsartan, with higher doses associated with lower rates of incident dementia [116].

The OSCAR study, evaluating the effect of 6 months of eprosartan therapy on cognitive function resulted in significant improvement on the overall mean Mini-Mental State Examination (MMSE) in patients with essential hypertension [121].

In another study, lack of significant cognitive decline was observed with telmisartan in patients with probable $\mathrm{AD}$ after 6 months of treatment, while patients treated with amlodipine showed a worsening on the AD Assessment Scale-Cognitive Subscale score. Regional cerebral blood flow detected on single-photon emission computed tomography (SPECT) was also improved in several cerebral regions with telmisartan use [122]. 
The ongoing SARTAN-AD study, sponsored by the Alzheimer's Drug Discovery Foundation Canada, will evaluate the amount of brain atrophy after telmisartan treatment versus perindopril in patients with probable AD and mild to moderate hypertension [123].

\section{Conclusions}

With an aim to prevent $\mathrm{CV}$ events, primarily telmisartan and eventually losartan are the ARBs of choice in patients with high $\mathrm{CV}$ risk and a general need for $\mathrm{CV}$ risk reduction. In the case of HF patients, losartan, candesartan or valsartan should be chosen. Valsartan should also be considered for patients with left ventricular dysfunction following a recent MI. For hypertensive patients at a higher risk of stroke, losartan should be the first therapeutic option; telmisartan, eprosartan and candesartan have also shown risk reduction in this subpopulation. In the case of patients with a history of AF, the use of telmisartan can be recommended; losartan, candesartan and valsartan have shown a reduced incidence of new-onset AF; therefore their use may also be beneficial in patients at risk of AF.

In patients at risk of developing DM, many ARBs (such as losartan, telmisartan, irbesartan, candesartan and valsartan) have shown protective properties. Telmisartan has also improved several indicators in those with increased IR or DM (e.g., fasting plasma glucose and insulin levels); therefore its use can definitely be recommended in diabetic patients. In terms of cardioprotection in patients with DM, both telmisartan and valsartan have shown lower risk of admission to hospital due to MI, stroke or HF.

Based on the results of robust, well-designed trials, losartan and irbesartan have been indicated for the treatment of diabetic nephropathy and should be the ARBs of choice in these patients. Valsartan and candesartan have also shown some benefits in this population. According to a recent metaanalysis, telmisartan may even be more beneficial in reducing proteinuria than other ARBs. Given the wide range of available ARBs, olmesartan use should be avoided in DM patients, especially in those with CKD, as some data suggest increased CV risk with its use in this population.

Due to its insulin-sensitizing properties, the choice of the PPAR $-\gamma$ agonist telmisartan should be considered in obese hypertensive patients showing signs of metabolic syndrome. Valsartan was also shown to improve insulin sensitivity in obese hypertensive patients.

In patients with higher uric acid levels, the ARB of choice should be losartan. Irbesartan may also have a protective effect at therapeutic doses. Telmisartan is a neutral agent regarding uric acid excretion, while candesartan, olmesartan and valsartan may increase the risk of hyperuricemia.
The most investigated ARBs in patients with ED are valsartan, irbesartan and losartan; the latter two have also been studied in coexisting metabolic syndrome and DM. Irbesartan also showed beneficial effects in patients with ED after prostatectomy. Telmisartan did not show any influence on ED.

Several ARBs have shown a beneficial effect on cognitive function in animal studies; in human trials, ARBs reduced the incidence as well as progression of $\mathrm{AD}$ and dementia. Further research is needed in order to establish their role in the treatment of diseases causing cognitive impairment. Meanwhile, their use may be beneficial and can be recommended in hypertensive patients at risk of dementia.

\section{Compliance with Ethical Standards}

Conflict of interest The author has no conflicts of interest that might be relevant to the contents of this manuscript.

Open Access This article is distributed under the terms of the Creative Commons Attribution-NonCommercial 4.0 International License (http://creativecommons.org/licenses/by-nc/4.0/), which permits any noncommercial use, distribution, and reproduction in any medium, provided you give appropriate credit to the original author(s) and the source, provide a link to the Creative Commons license, and indicate if changes were made.

\section{References}

1. James PA, Oparil S, Carter BL, et al. 2014 Evidence-based guideline for the management of high blood pressure in adults. Report from the panel members appointed to the Eighth Joint National Committee (JNC 8). JAMA. 2014;311(5):507-20.

2. The Task Force for the management of arterial hypertension of the European Society of Hypertension and of the European Society of Cardiology. ESH/ESC Guidelines for the management of arterial hypertension. J Hypertens. 2013;2013(31):1281-357.

3. Rosendorff $\mathrm{C}$, et al. Treatment of hypertension in patients with coronary artery disease: a scientific statement from the American Heart Association, American College of Cardiology, and American Society of Hypertension. J Am Soc Hypertens. 2015;9(6):453-98.

4. Li Robert. Cardiovascular diseases: from molecular pharmacology to evidence-based therapeutics. Hoboken: Wiley.; 2015.

5. Negro R. Endothelial effects of antihypertensive treatment: focus on irbesartan. Vasc Health Risk Manag. 2008;4(1):89-101.

6. Aoyama T, Minatoguchi S. The effect of ARB on prevention of atherosclerosis. Nihon Rinsho. 2011;69(1):92-9.

7. Carey RM. Role of the angiotensin type 2 receptor in the regulation of blood pressure and renal function. Hypertension. 2000;35[part 2]:155-63.

8. Blood Pressure Lowering Treatment Trialists' Collaboration. Effects of different blood-pressure-lowering regimens on major cardiovascular events: results of prospectively-designed overviews of randomised trials. Lancet. 2003;362(9395):1527-35.

9. https://www.medicines.org.uk/emc/

10. The ONTARGET Investigators. Telmisartan, ramipril, or both in patients at high risk for vascular events. $\mathrm{N}$ Engl $\mathrm{J}$ Med. 2008;358:1547-59. 
11. TRANSCEND Investigators. Effects of the angiotensin-receptor blocker telmisartan on cardiovascular events in high-risk patients intolerant to angiotensin-converting enzyme inhibitors: a randomised controlled trial. Lancet. 2008;372(9644):1174-83.

12. Ripley TL. The power to TRANSCEND. Lancet. 2008;372(9644):1128-30.

13. Scheen AJ. Clinical study of the month. The LIFE study: cardiovascular protection of hypertensive patients by losartan. Rev Med Liege. 2002;57(4):240-4.

14. Piérard L. Clinical study of the month. Effects of valsartan in chronic heart failure: the VAL-HeFT study. Rev Med Liege. 2002;57(1):57-9.

15. McKelvie RS, et al. Comparison of candesartan, enalapril, and their combination in congestive heart failure randomized evaluation of strategies for left ventricular dysfunction (RESOLVD) pilot study. Circulation. 1999;100:1056-64.

16. Pfeffer MA, et al. Effects of candesartan on mortality and morbidity in patients with chronic heart failure: the CHARMOverall programme. Lancet. 2003;362(9386):759-66.

17. Pitt B, et al. Randomised trial of losartan versus captopril in patients over 65 with heart failure (Evaluation of Losartan in the Elderly Study, ELITE). Lancet. 1997;349(9054):747-52.

18. Pitt B, et al. Effect of losartan compared with captopril on mortality in patients with symptomatic heart failure: randomised trial-the Losartan Heart Failure Survival Study ELITE II. Lancet. 2000;355(9215):1582-7.

19. Konstam MA, et al. Effects of high-dose versus low-dose losartan on clinical outcomes in patients with heart failure (HEAAL study): a randomised, double-blind trial. Lancet. 2009;374(9704):1840-8.

20. Solomon SD, et al. Changes in ventricular size and function in patients treated with valsartan, captopril, or both after myocardial infarction. Circulation. 2005;111:3411-9.

21. Noro T, et al. Initial reduction of oxidative stress by angiotensin receptor blocker contributes long term outcomes after percutaneous coronary intervention. Am J Cardiovasc Dis. 2014;4(4):159-67.

22. Foulquier $\mathrm{S}$, et al. Impact of telmisartan on cardiovascular outcome in hypertensive patients at high risk: a Telmisartan Randomised AssessmeNt Study in ACE iNtolerant subjects with cardiovascular Disease subanalysis. J Hypertens. 2014;32(6):1334-41.

23. Kjeldsen SE, et al. The effects of losartan compared to atenolol on stroke in patients with isolated systolic hypertension and left ventricular hypertrophy. The LIFE study. J Clin Hypertens (Greenwich). 2005;7(3):152-8.

24. Yusuf S, et al. Telmisartan to prevent recurrent stroke and cardiovascular events. N Engl J Med. 2008;359(12):1225-37.

25. Diener HC. Preventing stroke: the PRoFESS, ONTARGET, and TRANSCEND trial programs. J Hypertens Suppl. 2009;27(5):S31-6.

26. Papademetriou V, et al. Stroke prevention with the angiotensin II type 1-receptor blocker candesartan in elderly patients with isolated systolic hypertension: the Study on Cognition and Prognosis in the Elderly (SCOPE). J Am Coll Cardiol. 2004;44(6):1175-80.

27. Schrader J, et al. The ACCESS Study. Evaluation of acute candesartan cilexetil therapy in stroke survivors. Stroke. 2003;34:1699-703.

28. Schrader J, et al. Morbidity and mortality after stroke, eprosartan compared with nitrendipine for secondary prevention: principal results of a prospective randomized controlled study (MOSES). Stroke. 2005;36:1218-26.

29. Wachtell K, et al. Angiotensin II receptor blockade reduces new-onset atrial fibrillation and subsequent stroke compared to atenolol: the Losartan Intervention for End Point Reduction in Hypertension (LIFE) study. J Am Coll Cardiol. 2005;45:712-9.

30. Ducharme A, et al. Prevention of atrial fibrillation in patients with symptomatic chronic heart failure by candesartan in the Candesartan in Heart failure: Assessment of Reduction in Mortality and morbidity (CHARM) program. Am Heart J. 2006;152:86-92.

31. Schmieder R, et al. Reduced incidence of new-onset atrial fibrillation with angiotensin II receptor blockade: the VALUE trial. J Hypertens. 2008;26:403-11.

32. Maggioni A, et al. Valsartan reduces the incidence of atrial fibrillation in patients with heart failure: results from the Valsartan Heart Failure Trial (Val-HeFT). Am Heart J. 2005;149:548-57.

33. Takii E, et al. Beneficial effects of losartan for prevention of paroxysmal atrial fibrillation in patients with sick sinus syndrome: analysis with memory function of pacemaker. Heart Vessels. 2015 [Epub ahead of print].

34. Fogari R, et al. Effect of telmisartan and ramipril on atrial fibrillation recurrence and severity in hypertensive patients with metabolic syndrome and recurrent symptomatic paroxysmal and persistent atrial fibrillation. J Cardiovasc Pharmacol Ther. 2012;17(1):34-43.

35. Fogari R, et al. Effect of telmisartan on paroxysmal atrial fibrillation recurrence in hypertensive patients with normal or increased left atrial size. Clin Cardiol. 2012;35(6):359-64.

36. Galzerano D, et al. A multicentre, randomized study comparing efficacy of telmisartan versus carvedilol in preventing atrial fibrillation recurrence in hypertensive patients. Circulation. 2007;116(Suppl 2):556-7.

37. Fogari R, Mugellini A, Destro M, et al. Losartan and prevention of atrial fibrillation recurrence in hypertensive patients. J Cardiovasc Pharmacol. 2006;47(1):46-50.

38. Fogari R, Zoppi A, Mugellini A, et al. Comparative evaluation of effect of valsartan/amlodipine and atenolol/amlodipine combinations on atrial fibrillation recurrence in hypertensive patients with type 2 diabetes mellitus. J Cardiovasc Pharmacol. 2008;51(3):217-22.

39. Fogari R, et al. Effect of valsartan and ramipril on atrial fibrillation recurrence and $\mathrm{P}$-wave dispersion in hypertensive patients with recurrent symptomatic lone atrial fibrillation. Am J Hypertens. 2008;21(9):1034-9.

40. Disertori M, et al. Valsartan for prevention of recurrent atrial fibrillation. N Engl J Med. 2009;360(16):1606-17.

41. Madrid AH, et al. Use of irbesartan to maintain sinus rhythm in patients with long-lasting persistent atrial fibrillation. a prospective and randomized study. Circulation. 2002;106:331-6.

42. Yusuf $\mathrm{S}$, et al. Irbesartan in patients with atrial fibrillation. N Engl J Med. 2011;364(10):928-38.

43. Qi WW, et al. Upstream therapeutic strategies of valsartan and fluvastatin on hypertensive patients with non-permanent atrial fibrillation (VF-HT-AF): study protocol for a randomized controlled trial. Trials. 2015;16(1):336.

44. Luther JM, Brown NJ. The renin-angiotensin-aldosterone system and glucose homeostasis. Trends Pharmacol Sci. 2011;32(12):734-9.

45. van der Zijl NJ, et al. Does interference with the renin-angiotensin system protect against diabetes? Evidence and mechanisms. Diabetes Obes Metab. 2012;14(7):586-95.

46. Kintscher U, et al. Effect of high-dose valsartan on inflammatory and lipid parameters in patients with type 2 diabetes and hypertension. Diabetes Res Clin Pract. 2010;89(3):209-15.

47. Chang $\mathrm{CH}$, et al. Different angiotensin receptor blockers and incidence of diabetes: a nationwide population-based cohort study. Cardiovasc Diabetol. 2014;13:91. 
48. Zhao M, et al. Azilsartan treatment improves insulin sensitivity in obese spontaneously hypertensive Koletsky rats. Diabetes Obes Metab. 2011;13(12):1123-9.

49. https://www.clinicaltrials.gov/ct2/show/NCT02079805

50. Suksomboon N, et al. Systematic review of the effect of telmisartan on insulin sensitivity in hypertensive patients with insulin resistance or diabetes. J Clin Pharm Ther. 2012;37(3):319-27.

51. Tocci G. Angiotensin-converting enzyme inhibitors, angiotensin II receptor blockers and diabetes: a meta-analysis of placebocontrolled clinical trials. Am J Hypertens. 2011;24(5):582-90.

52. Andraws R, et al. Effect of inhibition of the renin-angiotensin system on development of type 2 diabetes mellitus (meta-analysis of randomized trials). Am J Cardiol. 2007;99(7):1006-12.

53. Cheung BMY, et al. Meta-analysis of large outcome trials of angiotensin receptor blockers in hypertension. J Hum Hypertens. 2006;20:37-43.

54. Kurtz TW, Klein U. Next generation multifunctional angiotensin receptor blockers. Hypertens Res. 2009;32:826-34.

55. Antoniou $\mathrm{T}$, et al. Comparative effectiveness of angiotensinreceptor blockers for preventing macrovascular disease in patients with diabetes: a population-based cohort study. CMAJ. 2013;185(12):1035-41.

56. Remuzzi G, Bertani T. Pathophysiology of progressive nephropathies. N Engl J Med. 1998;339:1448-56.

57. Ruggenenti $P$, et al. Progression, remission, regression of chronic renal diseases. Lancet. 2001;357:1601-8.

58. Palmer SC, et al. Comparative efficacy and safety of blood pressure-lowering agents in adults with diabetes and kidney disease: a network meta-analysis. Lancet. 2015;385(9982):2047-56.

59. Ogawa $S$, et al. Angiotensin II type 1 receptor blockers reduce urinary oxidative stress markers in hypertensive diabetic nephropathy. Hypertension. 2006;47:699-705.

60. Brenner BM, et al. Effects of losartan on renal and cardiovascular outcomes in patients with type 2 diabetes and nephropathy. N Engl J Med. 2001;345:861-9.

61. Lewis EJ, et al. Renoprotective effect of the angiotensin-receptor antagonist irbesartan in patients with nephropathy due to type 2 diabetes. N Engl J Med. 2001;345(12):851-60.

62. Andersen $\mathrm{S}$, et al. Kidney function during and after withdrawal of long-term irbesartan treatment in patients with type 2 diabetes and microalbuminuria. Diabetes Care. 2003;26(12):3296-302.

63. Takagi H, et al. Effects of telmisartan on proteinuria or albuminuria: a meta-analysis of randomized trials. Int $\mathrm{J}$ Cardiol. 2013;167(4):1443-9.

64. Yasuda T, et al. Effects of valsartan on progression of kidney disease in Japanese hypertensive patients with advanced, predialysis, chronic kidney disease: Kanagawa Valsartan Trial (KVT). Hypertens Res. 2013;36(3):240-6.

65. Viberti G, Wheeldon NM. MicroAlbuminuria Reduction with VALsartan (MARVAL) Study Investigators: microalbuminuria reduction with valsartan in patients with type 2 diabetes mellitus: a blood pressure-independent effect. Circulation. 2002;106:672-8.

66. Zhang X, et al. Efficacy and safety of valsartan in reducing blood pressure and albuminuria in Chinese patients with essential hypertension: a multicenter prospective open-label observational study. Curr Med Res Opin. 2012;28:1677-84.

67. Mogensen CE, et al. Randomised controlled trial of dual blockade of renin-angiotensin system in patients with hypertension, microalbuminuria, and non-insulin dependent diabetes: the candesartan and lisinopril microalbuminuria (CALM) study. BMJ. 2000;321(7274):1440-4.

68. Haller $\mathrm{H}$, et al. Olmesartan for the delay or prevention of microalbuminuria in type 2 diabetes. N Engl J Med. 2011;364:907-17.

69. Imai E, et al. Effects of olmesartan on renal and cardiovascular outcomes in type 2 diabetes with overt nephropathy: a multicentre, randomised, placebo-controlled study. Diabetologia. 2011;54(12):2978-86.

70. Padwal R, et al. Comparative effectiveness of olmesartan and other angiotensin receptor blockers in diabetes mellitus retrospective cohort study. Hypertension. 2014;63:977-83.

71. Hamamoto Yoshiyuki, Koshiyama Hiroyuki. ROADMAP and ORIENTAL trials: the re-emergence of J-curve ghost? Jpn Clin Med. 2011;2:25-8.

72. Huang Gao-Zhong, et al. Effects of telmisartan on insulin resistance and visceral fat distribution in Chinese hypertensive patients with obesity. Saudi Med J. 2011;32(10):1017-21.

73. Kadowaki T, Yamauchi T. Adiponectin and adiponectin receptors. Endocr Rev. 2005;26:439-51.

74. Padwal R, Laupacis A. Antihypertensive therapy and incidence of type 2 diabetes: a systematic review. Diabetes Care. 2004;27:247-55.

75. Takagi, et al. Telmisartan as a metabolic sartan: the first metaanalysis of randomized controlled trials in metabolic syndrome. J Am Soc Hypertens. 2013;7(3):229-35.

76. Mori Yutaka, et al. Influence of telmisartan on insulin response after glucose loading in obese patients with hypertension: ARB Trial of Hypertension in Obese Patients with Hyperinsulinemia Assessed by Oral Glucose Tolerance Test (ATHLETE). Adv Ther. 2011;28(8):698-706.

77. Chujo D, et al. Telmisartan treatment decreases visceral fat accumulation and improves serum levels of adiponectin and vascular inflammation markers in Japanese hypertensive patients. Hypertens Res. 2007;30:1205-10.

78. Murakami Kazutoshi, et al. The effects of telmisartan treatment on the abdominal fat depot in patients with metabolic syndrome and essential hypertension: Abdominal fat Depot Intervention Program of Okayama (ADIPO). Diab Vasc Dis Res. 2013;10(1):93-6.

79. Taksali SE, et al. High visceral and low abdominal subcutaneous fat stores in the obese adolescent: a determinant of an adverse metabolic phenotype. Diabetes. 2008;57:367-71.

80. Fogari R, et al. Comparative effects of telmisartan and eprosartan on insulin sensitivity in the treatment of overweight hypertensive patients. Horm Metab Res. 2009;41(12):893-8.

81. Brook RD, et al. Valsartan improves insulin sensitivity without altering vascular function in healthy overweight adults without the metabolic syndrome. Metab Syndr Relat Disord. 2007;5(3):255-61.

82. Goossen GH, et al. Valsartan improves adipose tissue function in humans with impaired glucose metabolism: a randomized placebo-controlled double-blind trial. PLoS One. 2012;7(6):e39930.

83. Park JB, Sung KC, Kang SM, et al. Safety and efficacy of fimasartan in patients with arterial hypertension (Safe-KanArb Study): an open-label observational study. Am J Cardiovasc Drugs. 2013;13(1):47-56.

84. Conde-Carmona I, Cardona E. Open label study of the efficacy and safety of fimasartan $60 \mathrm{mg}$ alone as initial treatment and its randomized escalation to fimasartan $120 \mathrm{mg}$ or fimasartan $60 \mathrm{mg}$ /hydrochlorothiazide $12.5 \mathrm{mg}$ in Mexican patients with essential hypertension grade 1 or 2. J Am Coll Cardiol. 2015;65(10_S). doi:10.1016/S0735-1097(15)61393-6.

85. Kim C, Kim MY, Kang DR, et al. The Efficacy of Fimasartan for Cardiovascular Events and Metabolic Syndrome (K-MetS Study): rationale, design and participant characteristics. Pulse. 2013;1(3-4):177-85.

86. Csiky B. The pleiotropic effects of losartan [Hungarian]. LAM. 2008;18(10):663-66.

87. Hainer BL, et al. Diagnosis, treatment, and prevention of gout. Am Fam Physician. 2014;90(12):831-6.

88. Iwanaga $\mathrm{T}$, et al. Concentration-dependent mode of interaction of angiotensin II receptor blockers with uric acid transporters. JPET. 2007;320:211-7. 
89. Fauvel JP, et al. Effects of losartan on renal function in patients with essential hypertension. J Cardiovasc Pharmacol. 1996;28:259-63.

90. Manolis AJ, et al. Effects of losartan and candesartan monotherapy and losartan/hydrochlorothiazide combination therapy in patients with mild to moderate hypertension: Losartan Trial Investigators. Clin Ther. 2000;22:1186-203.

91. Nakashima M, et al. Pilot study of the uricosuric effect of DuP753, a new angiotensin II receptor antagonist, in healthy subjects. Eur J Clin Pharmacol. 1992;42:333-5.

92. Ilson BE, et al. The effects of eprosartan, an angiotensin II AT1 receptor antagonist, on uric acid excretion in patients with mild to moderate essential hypertension. J Clin Pharmacol. 1998;38(5):437-41.

93. Nakamura M, et al. Effects of irbesartan on serum uric acid levels in patients with hypertension and diabetes. Clin Pharmacol. 2014;6:79-86.

94. Bohm M, Baumhakel M, Teo K, et al. Erectile dysfunction predicts cardiovascular events in high-risk patients receiving telmisartan, ramipril, or both: the Ongoing Telmisartan Alone and in combination with Ramipril Global Endpoint Trial/ Telmisartan Randomized Assessment Study in ACE intolerant subjects with cardiovascular Disease (ONTARGET/TRANSCEND) trials. Circulation. 2010;121(12):1439-46.

95. Doumas M, et al. Combined antihypertensive therapy and sexual dysfunction: terra incognita. Cardiology. 2013;125:232-4.

96. Becker AJ, et al. Possible role of bradykinin and angiotensin II in the regulation of penile erection and detumescence. Urology. 2001;57(1):193-8.

97. Viigimaa M, et al. (eds). Erectile dysfunction in hypertension and cardiovascular disease: a guide for clinicians. Berlin: Springer; 2014.

98. Nicolai MPJ, et al. A review of the positive and negative effects of cardiovascular drugs on sexual function: a proposed table for use in clinical practice. Neth Heart J. 2014;22:11-9.

99. Baumhakel M, et al. Improvement of endothelial function of the corpus cavernosum in apolipoprotein E knockout mice treated with irbesartan. J Pharmacol Exp Ther. 2008;327(3):692-8.

100. Shimizu S. Effect of an angiotensin II receptor blocker and a calcium channel blocker on hypertension associated penile dysfunction in a rat model. Biomed Res. 2014;35(3):215-21.

101. Della Chiesa A, et al. Sexual activity in hypertensive men. J Hum Hypertens. 2003;17(8):515-21.

102. Dusing R. Effect of the angiotensin II antagonist valsartan on sexual function in hypertensive men. Blood Press Suppl. 2003;2:29-34.

103. Fogari R, Zoppi A, Poletti L, et al. Sexual activity in hypertensive men treated with valsartan or carvedilol: a crossover study. Am J Hypertens. 2001;14(1):27-31.

104. Fogari R, et al. Effect of antihypertensive treatment with valsartan or atenolol on sexual activity and plasma testosterone in hypertensive men. Eur J Clin Pharmacol. 2002;58(3):177-80.

105. Chen Y, Cui S, Lin H, et al. Losartan improves erectile dysfunction in diabetic patients: a clinical trial. Int J Impot Res. 2012;24(6):217-20.
106. Llisterri JL, et al. Sexual dysfunction in hypertensive patients treated with losartan. Am J Med Sci. 2001;321(5):336-41.

107. Baumhäkel M, et al. Effect of irbesartan on erectile function in patients with hypertension and metabolic syndrome. Int J Impot Res. 2008;20(5):493-500.

108. Segal RL, et al. Irbesartan promotes erection recovery after nerve-sparing radical retropubic prostatectomy: a retrospective long-term analysis. BJU Int. 2012;110(11):1782-6.

109. Elias MF, Wolf PA, d'Agostino RB, et al. Untreated blood pressure levels is inversely related to cognitive functioning: the Framingham Study. Am J Epidemiol. 1993;138:353-64

110. Kilander L, Nyman H, Boberg M, et al. Hypertension is related to cognitive impairment: a 20-year follow-up of 999 men. Hypertension. 1998;31:780-6.

111. Launer LJ, Ross GW, Petrovitch H, et al. Midlife blood pressure and dementia: the Honolulu-Asia aging study. Neurobiol Aging. 2000;21:49-55.

112. Skoog I, Lernfelt B, Landahl S, et al. 15-year longitudinal study of blood pressure and dementia. Lancet. 1996;347:1141-5.

113. Tzourio C, Dufouil C, Ducimetiere P, et al. Cognitive decline in individuals with high blood pressure: a longitudinal study in the elderly. EVA Study Group. Neurology. 1999;53:1948-52.

114. Mielke MM, Rosenberg PB, Tschanz J, et al. Vascular factors predict rate of progression in Alzheimer disease. Neurology. 2007;69:1850-8.

115. Roselli F, Tartaglione B, Federico F, et al. Rate of MMSE score change in Alzheimer's disease: influence of education and vascular risk factors. Clin Neurol Neurosurg. 2009;111:327-30.

116. Li N-C, Lee A, Whitmer RA, et al. Use of angiotensin receptor blockers and risk of dementia in a predominantly male population: prospective cohort analysis. BMJ. 2010;340:b5465.

117. Ongali B, et al. Angiotensin II type 1 receptor blocker losartan prevents and rescues cerebrovascular, neuropathological and cognitive deficits in an Alzheimer's disease model. Neurobiol Dis. 2014;68:126-36.

118. Pelisch N, et al. Blockade of $\mathrm{AT}_{1}$ receptors protects the bloodbrain barrier and improves cognition in Dahl salt-sensitive hypertensive rats. Am J Hypertens. 2011;24(3):362-8.

119. Kishi Takuya, et al. Telmisartan protects against cognitive decline via up-regulation of brain-derived neurotrophic factor/ tropomyosin-related kinase B in hippocampus of hypertensive rats. J Cardiol. 2012;60:489-94.

120. Wincewicz D, Braszko JJ. Telmisartan attenuates cognitive impairment caused by chronic stress in rats. Pharmacol Rep. 2014;66:436-41.

121. Hanon O, et al. Effects of hypertension therapy based on eprosartan on systolic arterial blood pressure and cognitive function: primary results of the Observational Study on Cognitive function And Systolic Blood Pressure Reduction open-label study. J Hypertens. 2008;26(8):1642-50.

122. Kume Kazumasa, et al. Effects of telmisartan on cognition and regional cerebral blood flow in hypertensive patients with Alzheimer's disease. Geriatr Gerontol Int. 2012;12:207-14.

123. https://clinicaltrials.gov/ct2/show/NCT02085265?term=sartanad\&rank $=1$ 